\title{
Hosoya Polynomial, Wiener Index, Coloring and Planar of Annihilator Graph of $Z_{n}$
}

Mohammed S. Ahmed

mohammed.shaker.msa@gmail.com Department of Mathematics,

College of Computer Science and Mathematics,

University of Mosul, Mosul, Iraq
Akram S. Mohammed Nabeel E. Arif

Department of Mathematics, College of Computer Science and Mathematics, University of Tikrit, Tikrit, Iraq

Received on: 13/04/2020

Accepted on: 20/05/2020

\section{ABSTRACT}

Let $R$ be a commutative ring with identity. We consider $\Gamma_{\mathrm{B}}(R)$ an annihilator graph of the commutative ring $R$. In this paper, we find Hosoya polynomial, Wiener index, Coloring, and Planar annihilator graph of $Z_{n}$ denote $\Gamma_{\mathrm{B}}\left(Z_{n}\right)$, with $n=p^{m}$ or $n=$ $p^{m} q$, where $p, q$ are distinct prime numbers and $m$ is an integer with $m \geq 1$.

Keywords: Annihilator graph of ring, Zero-divisor graph, Hosoya polynomial, Wiener index, coloring graph and planar graph.

$$
\begin{aligned}
& \text { متعددة حدود هوسويا، دليل وينر، التلوين والاستواء لبيان تالف الحلقة الإبدالية }
\end{aligned}
$$

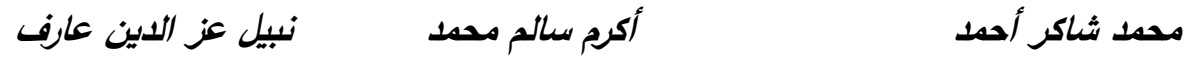

$$
\begin{aligned}
& \text { قسم الرياضيات، كلية علوم الحاسوب والرياضيات، قسم الرياضيات، كلية علوم الحاسوب والرياضيات، }
\end{aligned}
$$

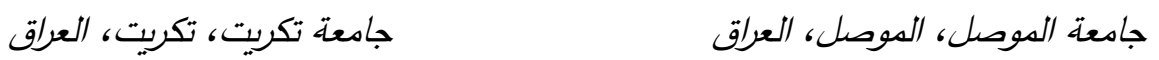

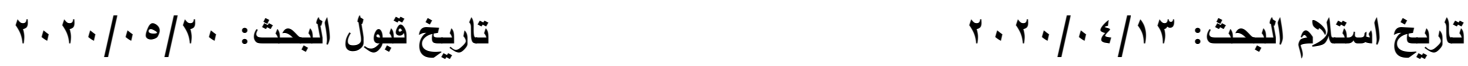

\section{الملنص}

لتكن R حلقة إبدالية بعنصر محايد. نعتبر (R) بلان $\Gamma_{\mathrm{B}}(R)$

هوسويا، دليل وينر، التلوين والاستواء لبيان تالف

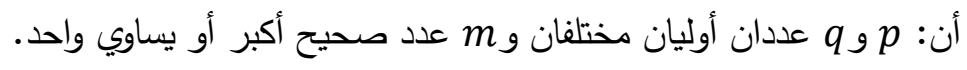

الكلمات المفتاحية: بيان تالف الحلقة، بيان القواسم الصفرية، متعددة حدود هوسويا، دليل وينر، تلوين البيان

$$
\text { واستواء البيان. }
$$

\section{Introduction}

Let $R$ be a commutative ring with identity the annihilator of $R$ is the set of all element $x \in R$ satisfy $\operatorname{ann}(R)=\{x \in R: x . y=0, \forall y \in R\}$ [6], and let $\operatorname{ann}(R)$ be the set of all annihilator in $R$. We consider a simple graph $\Gamma_{\mathrm{B}}(R)$ to $R$ with vertices $\operatorname{ann}(R)$, for every two distinct vertices $x, y$ are adjacent if and only if $\{x . y=$ $0: x, y \in \operatorname{ann}(R)\}$, and let $Z(R)$ be the set of all zero-divisors in $R$, and $Z(R)^{*}$ is the set of all non-zero zero-divisors in it. A simple graph $\Gamma(R)$ with vertices $Z(R)^{*}$, for every two distinct vertices $x, y$ are adjacent if and only if $\left\{x . y=0: x, y \in Z(R)^{*}\right\}$. 
The notion of an annihilator graph of a commutative ring was first introduced in 1988 by Beck [5], where he was interested in colorings, this investigation was then continued by Anderson and Nasser [3] zero-divisor graph of a commutative ring, further that Anderson and Livingston [2]. They denoted that by $\Gamma(R)$. It is clear that from Beck's definition of annihilator graph of a commutative ring and Anderson's definition of a zero-divisor graph of a commutative ring can be defined Annihilator graph of a commutative ring can be defined $\Gamma_{\mathrm{B}}(R)=\left(\left(\Gamma(R) \cup\left\{\operatorname{ann}\left(R^{*}\right)-Z(R)^{*}\right\}\right)+k_{1}\right)$. Such that: $\Gamma(R)$ zero-divisor graph of the ring, $\operatorname{ann}\left(R^{*}\right)$ set of all vertices in $R$ non-zero, $Z(R)^{*}$ set of all non-zero zero-divisors in $R$ and $k_{1}=0$.

A graph $G$ is called a connected graph if there is at least one path between any pair of vertices in $G$, otherwise it is called disconnected [7]. For vertices $x, y$ of $G$, let $d(x, y)$ be the length of the shortest path from $x$ to $y$ (and it is called distance between two vertices $x, y$ in $G$ ). The maximum distance between any two vertices $x, y$ in $G$ is called the diameter graph $G$ [7], that is $\operatorname{diam}(G)=\max _{x, y \in V(G)}\{d(x, y)\}$, where $V(G)$ is the set of all vertices of $G$. A graph $G$ is complete if every two of its vertices are adjacent, so the complete graph of order $n$ is denoted by $k_{n}$. If the vertex set of a graph $G$ can be split into two disjoint sets $A$ and $B$ (such that the induced subgraph that generated by either $A$ or $B$ is null graph), then we said $G$ is a bipartite graph. This graph is also said to be a complete bipartite graph is a bipartite graph in graph if each vertex in the set $A$ has joined to every vertex in the set $B$ with just one edge.

Hosoya polynomial of the graph $G$ is defined by $H(G ; x)=\sum_{k=0}^{\operatorname{diam}(G)} d(G, k) x^{k}$, where $d(G, k)$ is the number of pairs of vertices of a graph $G$, that are at distance $k$ apart, for $k=0,1,2, \ldots, \operatorname{diam}(G)$. The Wiener index of $G$ is defined as the sum of all distances between vertices of the graph $G$, and denoted by $W(G)$, we can also find this index by differentiating Hosoya polynomial with respect to $x$ at $x=1$, by symbols we can write: $W(G)=\left.\frac{d}{d x} H(G ; x)\right|_{x=1}$, See $[8,12]$.

Let $\mathcal{X}(G)$ denote the chromatic number of vertices, i.e., the minimal number of colors, which can be assigned to the vertices of $G$ in such a way that every two adjacent vertices have different colors [7]. We let $\bar{X}(G)$ denote the chromatic number of edges, i.e., the minimal number of colors, which can be assigned to the edges of $G$ in such a way that every two adjacent edges have different colors [7]. And last we assumed $f(G)$ denote the chromatic number of faces, i.e., the minimal number of colors, that can be assigned to the faces of planar graph $G$ in such a way that every two adjacent faces have different colors [7]. A planar graph $G$ is a graph that can be drawn in the plane without crossings for any two edges in $G$ [7]. There are many studies in the graph properties and commutative ring. See [1],[4],[10]\&[11].

\section{Some Properties of Graph $\Gamma_{B}\left(Z_{p^{m}}\right)$}

We will start this item by a lemma.

Lemma 2.1: The vertex (0) connect with every vertex of the graph $\Gamma_{\mathrm{B}}\left(Z_{n}\right)$.

Proof: Since 0. $a=0, \forall a \in Z_{n}$, so it is the vertex (0) connect with every vertex of the graph $\Gamma_{\mathrm{B}}\left(Z_{n}\right)$.

Lemma 2.2 [7]: Let $G$ be a connected graph of order $p$, then:

$$
\sum_{k=0}^{\operatorname{diam}(G)} d(G, k)=\left(\begin{array}{c}
p+1 \\
2
\end{array}\right)=\frac{1}{2} p(p+1) .
$$


Theorem 2.3: The Hosoya polynomial of graph $\Gamma_{\mathrm{B}}\left(Z_{p^{m}}\right)$ where $p$ is a prime number and $m$ is an integer with $m \geq 1$.

$$
\begin{aligned}
H\left(\Gamma_{\mathrm{B}}\left(Z_{p^{m}}\right) ; x\right)=p^{m}+\frac{1}{2}\left[(m+1) p^{m}\right. & \left.-m p^{m-1}-p^{\left\lfloor\frac{m}{2}\right\rfloor}\right] x \\
& +\frac{1}{2}\left[p^{2 m}-(m+2) p^{m}+m p^{m-1}+p^{\left\lfloor\frac{m}{2}\right\rfloor}\right] x^{2} .
\end{aligned}
$$

Proof: From the definition of the graph $\Gamma_{\mathrm{B}}(R)$, since the vertex $(0)$ connect with every vertex of the graph $\Gamma_{\mathrm{B}}\left(Z_{n}\right)$, so the order of the graph $\Gamma_{\mathrm{B}}\left(Z_{n}\right)$ which represents absolute term of Hosoya polynomial of the graph $\Gamma_{\mathrm{B}}\left(Z_{p^{m}}\right)$.

Now, we find the coefficient of $x$ that represent size of the graph $\Gamma_{\mathrm{B}}\left(Z_{p^{m}}\right)$ using the definition of the graph $\Gamma_{\mathrm{B}}(R)$ is the sum of $\left(p^{m}-1\right)$ of the edges (since the vertex (0) connect with every vertex the graph $\Gamma_{\mathrm{B}}\left(Z_{p^{m}}\right)$ from the Lemma (2.1), with $a_{1}$ of the graph $\Gamma\left(Z_{p^{m}}\right)[9]$ where as $\left(a_{1}=\frac{1}{2}\left[(m-1) p^{m}-m p^{m-1}-p^{\left.\mid \frac{m}{2}\right\rfloor}+2\right]\right)$ so we get.

$$
\begin{aligned}
a_{1}+\left(p^{m}-1\right) & =\frac{1}{2}\left[(m-1) p^{m}-m p^{m-1}-p^{\left\lfloor\frac{m}{2}\right\rfloor}+2\right]+\left(p^{m}-1\right) \\
& =\frac{1}{2}\left[(m+1) p^{m}-m p^{m-1}-p^{\left\lfloor\frac{m}{2}\right\rfloor}\right] .
\end{aligned}
$$

Now, we find the coefficient of $x^{2}$ as the diameter of the graph $\Gamma_{\mathrm{B}}\left(Z_{p^{m}}\right)$ is two from the Lemma (2.1) and using Lemma (2.2) so we get:

$$
\begin{aligned}
& \sum_{k=0}^{\operatorname{diam}\left(\Gamma_{\mathrm{B}}\left(Z_{\left.p^{m}\right)}\right)\right.} d\left(\Gamma_{\mathrm{B}}\left(Z_{p^{m}}\right), k\right)=\left(\begin{array}{c}
p^{m}+1 \\
2
\end{array}\right) \\
& \Rightarrow \frac{p^{m}\left(p^{m}+1\right)}{2}=d\left(\Gamma_{\mathrm{B}}\left(Z_{p^{m}}\right), 0\right)+d\left(\Gamma_{\mathrm{B}}\left(Z_{p^{m}}\right), 1\right)+d\left(\Gamma_{\mathrm{B}}\left(Z_{p^{m}}\right), 2\right) \\
& d\left(\Gamma_{\mathrm{B}}\left(Z_{p^{m}}\right), 2\right)=\frac{p^{m}\left(p^{m}+1\right)}{2}-d\left(\Gamma_{\mathrm{B}}\left(Z_{p^{m}}\right), 0\right)-d\left(\Gamma_{\mathrm{B}}\left(Z_{p^{m}}\right), 1\right) \\
& =\frac{p^{m}\left(p^{m}+1\right)}{2}-p^{m}-\frac{1}{2}\left[(m+1) p^{m}-m p^{m-1}-p^{\left\lfloor\frac{m}{2}\right\rfloor}\right] \\
& =\frac{1}{2}\left[p^{2 m}+p^{m}-2 p^{m}-m p^{m}-p^{m}+m p^{m-1}+p^{\left[\frac{m}{2}\right\rfloor}\right] \\
& =\frac{1}{2}\left[p^{2 m}-(m+2) p^{m}+m p^{m-1}+p^{\left\lfloor\frac{m}{2}\right\rfloor}\right] \text {. } \\
& \therefore H\left(\Gamma_{\mathrm{B}}\left(Z_{p^{m}}\right) ; x\right)=p^{m}+\frac{1}{2}\left[(m+1) p^{m}-m p^{m-1}-p^{\left[\frac{m}{2}\right]}\right] x \\
& +\frac{1}{2}\left[p^{2 m}-(m+2) p^{m}+m p^{m-1}+p^{\left\lfloor\frac{m}{2}\right\rfloor}\right] x^{2} .
\end{aligned}
$$

Corollary 2.4: The Wiener index of graph $\Gamma_{\mathrm{B}}\left(Z_{p^{m}}\right)$ where $p$ is prime number and $m$ is an integer with $m \geq 1$.

$$
W\left(\Gamma_{\mathrm{B}}\left(Z_{p^{m}}\right)\right)=\frac{1}{2}\left[2 p^{2 m}-(m+3) p^{m}+m p^{m-1}+p^{\left\lfloor\frac{m}{2}\right\rfloor}\right] .
$$

Proof: Since wiener index is the first derivative polynomial of Hosoya after compensation for a value $x=1$ so we get:

$$
\begin{aligned}
\because W\left(\Gamma_{B}\left(Z_{p^{m}}\right)\right) & =\left.\frac{d}{d x} H\left(\Gamma_{B}\left(Z_{p^{m}}\right) ; x\right)\right|_{x=1} \\
\therefore W\left(\Gamma_{B}\left(Z_{p^{m}}\right)\right) & =\frac{d}{d x}\left(p^{m}+\frac{1}{2}\left[(m+1) p^{m}-m p^{m-1}-p^{\left\lfloor\frac{m}{2}\right)}\right] x\right. \\
& \left.+\frac{1}{2}\left[p^{2 m}-(m+2) p^{m}+m p^{m-1}+p^{\left\lfloor\frac{m}{2}\right\rfloor}\right] x^{2}\right)\left.\right|_{x=1} \\
& =0+\frac{1}{2}\left[(m+1) p^{m}-m p^{m-1}-p^{\left\lfloor\frac{m}{2}\right\rfloor}\right]
\end{aligned}
$$




$$
\begin{aligned}
+\left.\left[p^{2 m}-(m+2) p^{m}+m p^{m-1}+p^{\left.\mid \frac{m}{2}\right\rfloor}\right] x\right|_{x=1} \\
=\frac{1}{2}\left[m p^{m}+p^{m}-m p^{m-1}-p^{\left.\mid \frac{m}{2}\right\rfloor}+2 p^{2 m}-2 m p^{m}-4 p^{m}\right. \\
\left.+2 m p^{m-1}+2 p^{\left\lfloor\frac{m}{2}\right\rfloor}\right] \\
=\frac{1}{2}\left[2 p^{2 m}-(m+3) p^{m}+m p^{m-1}+p^{\left\lfloor\frac{m}{2}\right\rfloor}\right] .
\end{aligned}
$$

Example 1: The Hosoya polynomial and wiener index of graph $\Gamma_{\mathrm{B}}\left(Z_{16}\right)$.

The graph is clear $\Gamma_{\mathrm{B}}\left(Z_{16}\right)$ of formula $\Gamma_{\mathrm{B}}\left(Z_{p^{m}}\right)$, where $p=2$ and $m=4$.

$$
\begin{aligned}
& \because H\left(\Gamma_{\mathrm{B}}\left(Z_{p^{m}}\right) ; x\right)=p^{m}+\frac{1}{2}\left[(m+1) p^{m}-m p^{m-1}-p^{\left\lfloor\frac{m}{2}\right\rfloor}\right] x \\
& \therefore H\left(\Gamma_{\mathrm{B}}\left(Z_{16}\right) ; x\right)=16+22 x+98 x^{2} . \\
& \because W\left(\Gamma_{\mathrm{B}}\left(Z_{p^{m}}\right)\right)=\frac{1}{2}\left[2 p^{2 m}-(m+2) p^{m}+m p^{m-1}+p^{\left\lfloor\frac{m}{2}\right\rfloor}\right] x^{2} . \\
& \left.\left.\therefore \mathrm{W}\left(\Gamma_{\mathrm{B}}\left(\mathrm{Z}_{16}\right)\right)=218.3\right) p^{m}+m p^{m-1}+p^{\left.\mid \frac{m}{2}\right)}\right] .
\end{aligned}
$$

\section{Theorem 2.5: (Coloring of graph $\Gamma_{B}\left(Z_{p^{m}}\right)$ ).}

A- Chromatic number of vertices of the graph $\Gamma_{\mathrm{B}}\left(Z_{p^{m}}\right)= \begin{cases}p^{\frac{m-1}{2}}+1, & m \text { is an odd. } \\ p^{\frac{m}{2}} & , m \text { is an even. }\end{cases}$

B- Chromatic number of edges of the graph $\Gamma_{\mathrm{B}}\left(Z_{p^{m}}\right)$ is $p^{m}-1$.

Proof: A- Case 1: if $m$ is an odd.

Since the multiplication of the number $p^{\frac{m+1}{2}}$ by one of its complication $\left(2 p^{\frac{m+1}{2}}, 3 p^{\frac{m+1}{2}}, \ldots, p^{\frac{m-1}{2}} \cdot p^{\frac{m+1}{2}}=0\right)$, that the product is one of its complications of the number $p^{m}$ which is equal to (0) in the ring $Z_{\boldsymbol{p}^{m}}$. Or multiplication one complication of the number $p^{\frac{m+1}{2}}$ in another complication of the number $p^{\frac{m+1}{2}}$ that the product is one of the complications of the number $p^{m}$ which is equal to (0) in the ring $Z_{\boldsymbol{p}^{m}}$ as in the Figure (2.1).

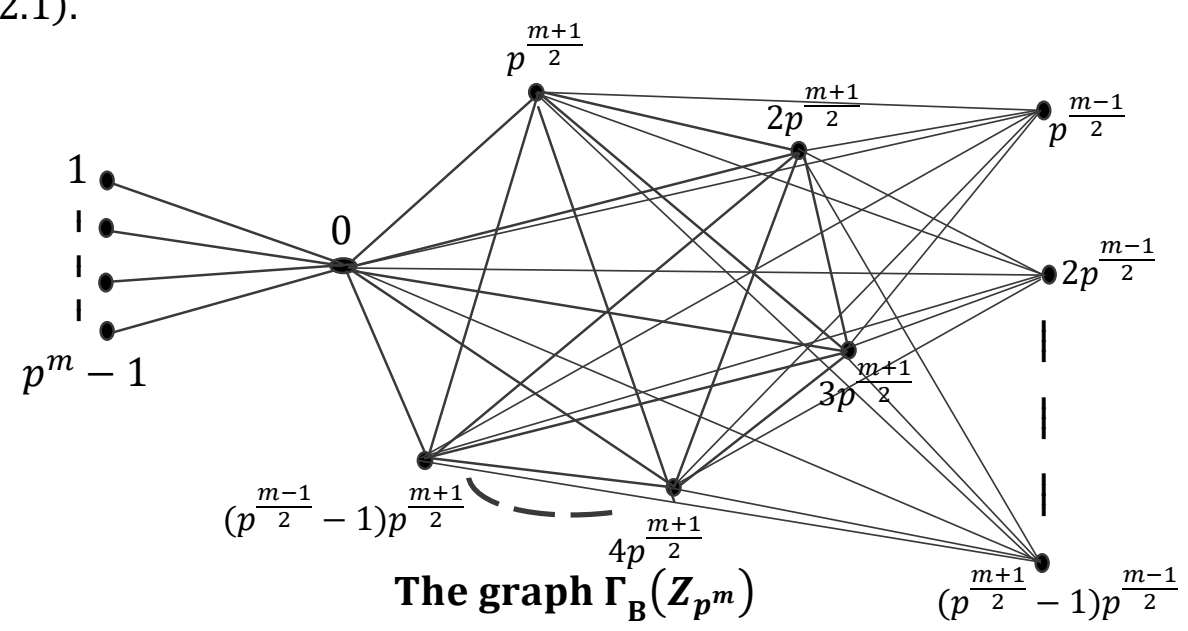

Fig (2.1) 
Clearly the complete graph $k_{p^{\frac{m-1}{2}}}$ be subgraph from the graph $\Gamma_{\mathrm{B}}\left(Z_{p^{m}}\right)$ (when $m$ is an odd), and also the multiplication of the number $p^{\frac{m-1}{2}}$ or one of its complications $\left(2 p^{\frac{m-1}{2}}, 3 p^{\frac{m-1}{2}}, \ldots, p^{\frac{m+1}{2}} \cdot p^{\frac{m-1}{2}}=0\right)$ by the number $p^{\frac{m+1}{2}}$ or one of its complications is the product $p^{m}$ or one of its complications which is equal to (0), in the ring $Z_{\boldsymbol{p}^{m}}$. And thus the complete graph $k_{p^{\frac{m-1}{2}}+1}$ is the largest complete subgraph that exist in the graph $\Gamma_{\mathrm{B}}\left(Z_{p^{m}}\right)$. And since the chromatic number of vertices of a complete graph $k_{p^{\frac{m-1}{2}}+1}$ is $\left(p^{\frac{m-1}{2}}+1\right)[7]$, so it is the chromatic number of the graph $\Gamma_{\mathrm{B}}\left(Z_{p^{m}}\right)$ is $\left(p^{\frac{m-1}{2}}+1\right)$ and also of vertices (when $m$ is an odd).

A- Case 2: if $m$ is an even:

Since the multiplication of the number $p^{\frac{m}{2}}$ by one of its complications $\left(2 p^{\frac{m}{2}}, 3 p^{\frac{m}{2}}, \ldots, p^{\frac{m}{2}} \cdot p^{\frac{m}{2}}=0\right)$, that the product is one of its complications of the number $p^{m}$ which is equal to (0) in the ring $Z_{p^{m}}$. Or multiplying one complication of the number $p^{\frac{m}{2}}$ in another complication of the number $p^{\frac{m}{2}}$, that the product is one of its complications of the number $p^{m}$, which is equal to (0) in the ring $Z_{p^{m}}$ as in the Figure (2.2).

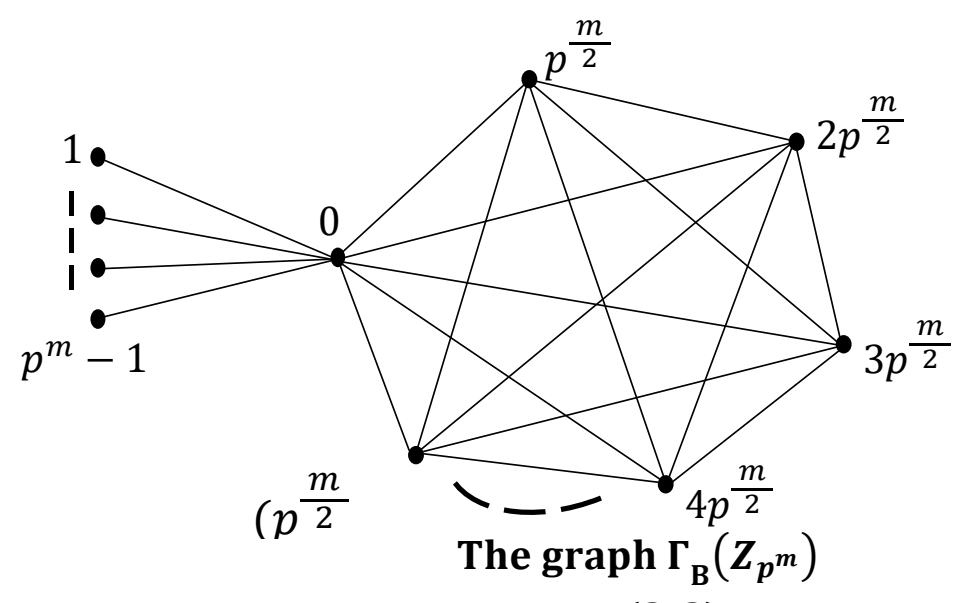

Fig (2.2)

Clearly the complete graph $k_{p^{\frac{m}{2}}}$ is the largest complete subgraph that exist in the graph $\Gamma_{\mathrm{B}}\left(Z_{p^{m}}\right)$ (when $m$ is an even). And since the chromatic number of vertices of a complete graph $\mathrm{k}_{p^{\frac{m}{2}}}$ is $p^{\frac{m}{2}}$ [7], so it is the chromatic number of the graph $\Gamma_{\mathrm{B}}\left(Z_{p^{m}}\right)$ is $p^{\frac{m}{2}}$ and also of vertices (when $m$ is an even).

B- From the Lemma (2.1) the vertex (0) connect with every vertex in the graph $\Gamma_{\mathrm{B}}\left(Z_{p^{m}}\right)$ then the degree of the vertex $(0)$ is $\left(p^{m}-1\right)$ so the chromatic number of the edges is $\left(p^{m}-1\right)$.

Theorem 2.6 [7]: (kuratowski's Theorem), The graph $G$ is planar if and only if it does not contain $G$ on subgraph that is homeomorphic to $k_{5}$ or $k_{3,3}$. 


\section{Theorem 2.7:}

a- The graph $\Gamma_{\mathrm{B}}\left(Z_{p^{m}}\right)$ contains a subgraph that is homeomorphic to $k_{p^{\frac{m-1}{2}}+1}$ and $k\left(p^{\frac{m+1}{2}}-p^{\frac{m-1}{2}}\right), p^{\frac{m-1}{2}}$ (when $m$ is an odd).

b- The graph $\Gamma_{\mathrm{B}}\left(Z_{p^{m}}\right)$ contains a subgraph that is homeomorphic to $k_{p^{\frac{m}{2}}}$ and $k\left(p^{\frac{m+2}{2}}-p^{\frac{m-2}{2}}\right), p^{\frac{m-2}{2}}$ (when $m$ is an even).

\section{Proof:}

a- From the Theorem (2.5-A-1) we get the first part of the Theorem directly.

Since the multiplication of the number $p^{\frac{m+1}{2}}$ or one of its complications $\left(2 p^{\frac{m+1}{2}}, 3 p^{\frac{m+1}{2}}, \ldots, p^{\frac{m-1}{2}} \cdot p^{\frac{m+1}{2}}=0\right)$ by number $p^{\frac{m-1}{2}}$ or one of its complications $\left(2 p^{\frac{m-1}{2}}, 3 p^{\frac{m-1}{2}}, \ldots, p^{\frac{m+1}{2}} \cdot p^{\frac{m-1}{2}}=0\right)$ be the product $p^{m}$ or one of its complications of the number $p^{m}$ which is equal to (0) in the ring $Z_{p^{m}}$. Thus, the graph $\Gamma_{\mathrm{B}}\left(Z_{p^{m}}\right)$ contains a subgraph homeomorphic with complete bipartite graph $k\left(p^{\frac{m+1}{2}}-p^{\frac{m-1}{2}}\right), p^{\frac{m-1}{2}}$ it is the largest complete bipartite graph there is in the graph $\Gamma_{\mathrm{B}}\left(Z_{p^{m}}\right)$ as in the Figure (2.3).

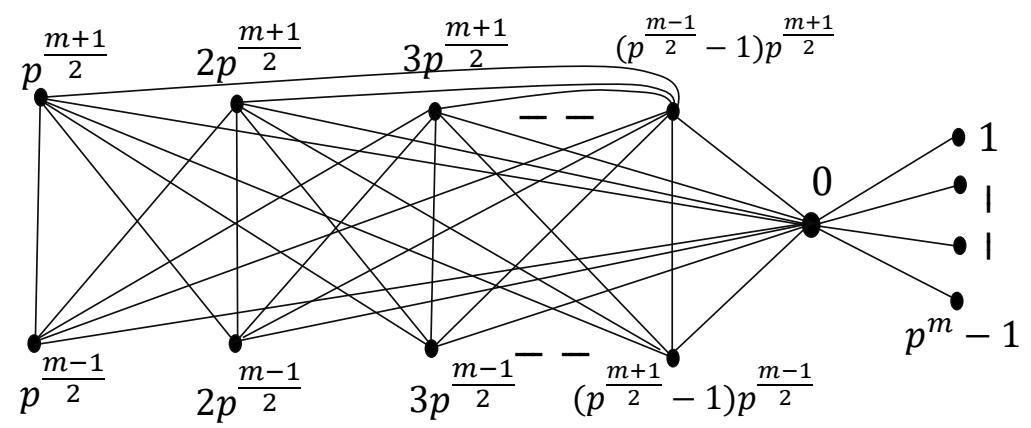

The graph $\Gamma_{B}\left(Z_{p^{m}}\right)$

\section{Fig (2.3)}

b- From the Theorem (2.5-A-2) we get the first part of the Theorem directly.

Since the multiplication of the number $p^{\frac{m+2}{2}}$ or one of its complications $\left(2 p^{\frac{m+2}{2}}, 3 p^{\frac{m+2}{2}}, \ldots, p^{\frac{m-2}{2}} \cdot p^{\frac{m+2}{2}}=0\right)$ by number $p^{\frac{m-2}{2}}$ or one of its complications $\left(2 p^{\frac{m-2}{2}}, 3 p^{\frac{m-2}{2}}, \ldots, p^{\frac{m+2}{2}} \cdot p^{\frac{m-2}{2}}=0\right)$ be the product $p^{m}$ or one of its complications of the number $p^{m}$ which is equal to (0) in the ring $Z_{p^{m}}$. Thus, the graph $\Gamma_{\mathrm{B}}\left(Z_{p^{m}}\right)$ contains a subgraph homeomorphic complete bipartite graph $k\left(p^{\frac{m+2}{2}}-p^{\frac{m-2}{2}}\right), p^{\frac{m-2}{2}}$ it is the largest complete bipartite graph there is in the graph $\Gamma_{\mathrm{B}}\left(Z_{p^{m}}\right)$ as in the Figure (2.4). 


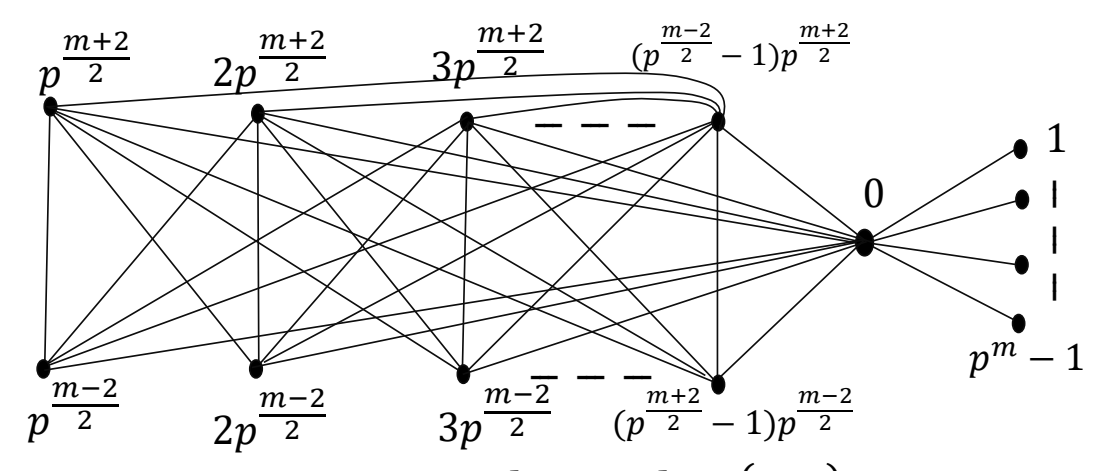

The graph $\Gamma_{\mathrm{B}}\left(Z_{p^{m}}\right)$

Fig (2.4)

\section{Remarks:}

1. From the Theorem (2.7-a), the only graphs $\Gamma_{\mathrm{B}}\left(Z_{p}\right)$ and $\Gamma_{\mathrm{B}}\left(Z_{8}\right)$ from the formula $\Gamma_{\mathrm{B}}\left(Z_{p^{m}}\right)$ when $m$ is an odd it does not contain subgraph homeomorphic $k_{5}$ or $k_{3,3}$ therefore it is planar graphs by kuratowski's Theorem.

2. From the Theorem (2.7-b), the only graphs $\Gamma_{\mathrm{B}}\left(Z_{4}\right), \Gamma_{\mathrm{B}}\left(Z_{9}\right)$ and $\Gamma_{\mathrm{B}}\left(Z_{16}\right)$ from the formula $\Gamma_{\mathrm{B}}\left(Z_{p^{m}}\right)$ when $m$ is an even it does not contain subgraph homeomorphic $\mathrm{k}_{5}$ or $\mathrm{k}_{3,3}$ therefore it is planar graphs by kuratowski's Theorem.

3. The only graphs $\Gamma_{\mathrm{B}}\left(Z_{4}\right), \Gamma_{\mathrm{B}}\left(Z_{8}\right), \Gamma_{\mathrm{B}}\left(Z_{9}\right), \Gamma_{\mathrm{B}}\left(Z_{16}\right)$ and $\Gamma_{\mathrm{B}}\left(Z_{p}\right)$ they are colorable for faces.

Example 2: The chromatic number of the graphs $\Gamma_{\mathrm{B}}\left(Z_{16}\right)$ and $\Gamma_{\mathrm{B}}\left(Z_{27}\right)$.

The graph is clear $\Gamma_{\mathrm{B}}\left(Z_{16}\right)$ of formula $\Gamma_{\mathrm{B}}\left(Z_{p^{m}}\right)$, where $p=2$ and $m=4$ and the graph is clear $\Gamma_{\mathrm{B}}\left(Z_{27}\right)$ of formula $\Gamma_{\mathrm{B}}\left(Z_{p^{m}}\right)$, where $p=3$ and $m=3$.

The chromatic number of vertices the graph $\Gamma_{\mathrm{B}}\left(\mathrm{Z}_{p^{m}}\right)$ is $p^{\frac{m}{2}}$ (when $m$ is an even).

$\therefore X\left(\Gamma_{\mathrm{B}}\left(Z_{16}\right)\right)=4$.

The chromatic number of edges the graph $\Gamma_{\mathrm{B}}\left(Z_{p^{m}}\right)$ is $p^{m}-1$.

$\therefore \bar{\chi}\left(\Gamma_{\mathrm{B}}\left(Z_{16}\right)\right)=15$.

From Theorem (2.7-b) we get the graph $\Gamma_{\mathrm{B}}\left(Z_{16}\right)$ contains a subgraph that is homeomorphic to $k_{4}$ and $k_{6,2}$ then the graph $\Gamma_{\mathrm{B}}\left(Z_{16}\right)$ it is planar by kuratowski's Theorem.

$\therefore f\left(\Gamma_{\mathrm{B}}\left(Z_{16}\right)\right)=3$.

The chromatic number of vertices the graph $\Gamma_{\mathrm{B}}\left(\mathrm{Z}_{p^{m}}\right)$ is $p^{\frac{m-1}{2}}+1$ (when $m$ is an odd).

$\therefore x\left(\Gamma_{\mathrm{B}}\left(Z_{27}\right)\right)=4$.

The chromatic number of edges the graph $\Gamma_{\mathrm{B}}\left(Z_{p^{m}}\right)$ is $p^{m}-1$.

$\therefore \bar{x}\left(\Gamma_{\mathrm{B}}\left(Z_{27}\right)\right)=26$.

From Theorem (2.7-a) we get the graph $\Gamma_{\mathrm{B}}\left(Z_{27}\right)$ contains a subgraph that is homeomorphic to $k_{6,3}$ then the graph $\Gamma_{\mathrm{B}}\left(Z_{27}\right)$ it is not planar by kuratowski's Theorem. 


\section{Some Properties of graph $\Gamma_{B}\left(Z_{p^{m} q}\right)$.}

Theorem 3.1: The Hosoya polynomial of graph $\Gamma_{\mathrm{B}}\left(Z_{p^{m}} q\right)$ where $p, q$ are distinct prime numbers and $m$ is an integer with $m \geq 1$.

$$
\begin{array}{r}
H\left(\Gamma_{\mathrm{B}}\left(Z_{p^{m} q}\right) ; x\right)=p^{m} q+\left(\frac{1}{2}[2 q(m p-m+p)-(m+1) p+m] p^{m-1}-\frac{1}{2} p^{\left\lfloor\frac{m}{2}\right\rfloor}\right) x \\
+\left(\frac{1}{2}\left[q\left(p^{m+1} q-3 p-2 m p+2 m\right)+(m+1) p-m\right] p^{m-1}+\frac{1}{2} p^{\left\lfloor\frac{m}{2}\right\rfloor}\right) x^{2} .
\end{array}
$$

Proof: From the definition of the graph $\Gamma_{\mathrm{B}}(R)$ since the vertex (0) connect with every vertex the graph $\Gamma_{\mathrm{B}}\left(Z_{p^{m} q}\right)$ so the order of the graph $\Gamma_{\mathrm{B}}\left(Z_{p^{m} q}\right)$ which represents absolute term Hosoya polynomial of graph $\Gamma_{\mathrm{B}}\left(Z_{p^{m} q}\right)$.

Now, we find the coefficient of $x$ that represent size of the graph $\Gamma_{B}\left(Z_{p^{m} q}\right)$ using the definition of the graph $\Gamma_{\mathrm{B}}(R)$ is the sum of $\left(Z_{p^{m}}-1\right)$ of the edges (since the vertex (0) connect with every vertex the graph $\Gamma_{\mathrm{B}}\left(Z_{p^{m} q}\right)$ from the Lemma (2.1), with $a_{1}$ of the graph $\Gamma\left(Z_{p^{m} q}\right)[9]$ where as $\left(a_{1}=\frac{1}{2}[2 m q(p-1)-(m+1) p+m] p^{m-1}-\right.$ $\left.p^{\left\lfloor\frac{m}{2}\right\rfloor}+1\right)$ so we get.

$$
\begin{aligned}
a_{1}+\left(p^{m} q-1\right) & =\frac{1}{2}[2 m q(p-1)-(m+1) p+m] p^{m-1}-p^{\left\lfloor\frac{m}{2}\right\rfloor}+1+p^{m} q-1 \\
& =\frac{1}{2}[2 \mathrm{q}(\mathrm{mp}-\mathrm{m}+\mathrm{p})-(\mathrm{m}+1) \mathrm{p}+\mathrm{m}] \mathrm{p}^{\mathrm{m}-1}-\frac{1}{2} \mathrm{p}^{\left\lfloor\frac{\mathrm{m}}{2}\right\rfloor} .
\end{aligned}
$$

Now, we find the coefficient of $x^{2}$ as the diameter of the graph $\Gamma_{\mathrm{B}}\left(Z_{p^{m} q}\right)$ is two from the Lemma (2.1) and using Lemma (2.2) so we get.

$$
\begin{aligned}
& \sum_{k=0}^{\operatorname{diam}\left(\Gamma_{\mathrm{B}}\left(Z_{p^{m}}\right)\right)} d\left(\Gamma_{\mathrm{B}}\left(Z_{p^{m} q}\right), k\right)=\left(\begin{array}{c}
p^{m} q+1 \\
2
\end{array}\right) \\
& \begin{aligned}
& \Rightarrow \frac{p^{m} q\left(p^{m} q+1\right)}{2}=d\left(\Gamma_{\mathrm{B}}\left(Z_{p^{m} q}\right), 0\right)+d\left(\Gamma_{\mathrm{B}}\left(Z_{p^{m} q}\right), 1\right)+d\left(\Gamma_{\mathrm{B}}\left(Z_{p^{m} q}\right), 2\right) \\
& d\left(\Gamma_{\mathrm{B}}\left(Z_{p^{m} q}\right), 2\right)=\frac{p^{m} q\left(p^{m} q+1\right)}{2}-d\left(\Gamma_{\mathrm{B}}\left(Z_{p^{m} q}\right), 0\right)-d\left(\Gamma _ { \mathrm { B } } \left(Z_{\left.\left.p^{m} q\right), 1\right)}\right.\right. \\
&=\frac{p^{m} q\left(p^{m} q+1\right)}{2}-p^{m} q-\left(\frac{1}{2}[2 q(m p-m+p)+m] p^{m-1}-\frac{1}{2} p^{\left\lfloor\frac{m}{2}\right\rfloor}\right) . \\
&=\frac{1}{2}\left[q\left(p^{m+1} q-3 p-2 m p+2 m\right)+(m+1) p-m\right] p^{m-1}+\frac{1}{2} p^{\left\lfloor\frac{m}{2}\right\rfloor} .
\end{aligned} \\
& \therefore H\left(\Gamma_{\mathrm{B}}\left(Z_{p^{m} q}\right) ; x\right)=p^{m} q+\left(\frac{1}{2}[2 q(m p-m+p)-(m+1) p+m] p^{m-1}-\frac{1}{2} p^{\left\lfloor\frac{m}{2}\right\rfloor}\right) x \\
& +\left(\frac{1}{2}\left[q\left(p^{m+1} q-3 p-2 m p+2 m\right)+(m+1) p-m\right] p^{m-1}+\frac{1}{2} p^{\left.\left[\frac{m}{2}\right\rfloor\right) x^{2} .}\right.
\end{aligned}
$$

Corollary 3.2: The Wiener index of $\Gamma_{\mathrm{B}}\left(Z_{p^{m} q}\right)$ where $p, q$ are distinct prime numbers and $m$ is an integer with $m \geq 1$.

$$
W\left(\Gamma_{\mathrm{B}}\left(Z_{p^{m} q}\right)\right)=\frac{1}{2}\left[2 q\left(p^{m+1} q-m p-2 p+m\right)+(m+1) p-m\right] p^{m-1}+\frac{1}{2} p^{\left\lfloor\frac{m}{2}\right\rfloor}
$$

Proof: Since wiener index is the first derivative polynomial of Hosoya after compensation for a value $x=1$ so we get:

$$
\begin{aligned}
& \because W\left(\Gamma_{\mathrm{B}}\left(Z_{p^{m}}\right)\right)=\left.\frac{d}{d x} H\left(\Gamma_{\mathrm{B}}\left(Z_{p^{m} q}\right) ; x\right)\right|_{x=1} \\
& \therefore W\left(\Gamma_{\mathrm{B}}\left(Z_{p^{m} q}\right)\right)=\frac{d}{d x}\left(p^{m} q+\left(\frac{1}{2}[2 q(m p-m+p)-(m+1) p+m] p^{m-1}-\frac{1}{2} p^{\left\lfloor\frac{m}{2}\right\rfloor}\right) x\right. \\
& \left.\quad+\left(\frac{1}{2}\left[q\left(p^{m+1} q-3 p-2 m p+2 m\right)+(m+1) p-m\right] p^{m-1}+\frac{1}{2} p^{\left\lfloor\frac{m}{2}\right\rfloor}\right) x^{2}\right)\left.\right|_{x=1}
\end{aligned}
$$




$$
\begin{gathered}
=\left(0+\left(\frac{1}{2}[2 q(m p-m+p)-(m+1) p+m] p^{m-1}-\frac{1}{2} p^{\left\lfloor\frac{m}{2}\right\rfloor}\right)\right. \\
+\left.\left(\left[q\left(p^{m+1} q-3 p-2 m p+2 m\right)+(m+1) p-m\right] p^{m-1}+\frac{1}{2} p^{\left\lfloor\frac{m}{2}\right\rfloor}\right) x\right|_{x=1} \\
=\frac{1}{2}\left[2 q\left(p^{m+1} q-m p-2 p+m\right)+(m+1) p-m\right] p^{m-1}+\frac{1}{2} p^{\left\lfloor\frac{m}{2}\right\rfloor} .
\end{gathered}
$$

Example 3: The Hosoya polynomial and wiener index of graph $\Gamma_{\mathrm{B}}\left(Z_{18}\right)$.

The graph is clear $\Gamma_{\mathrm{B}}\left(Z_{18}\right)$ of formula $\Gamma_{\mathrm{B}}\left(Z_{p^{m} q}\right)$, where $p=3, q=2$ and $m=2$.

$$
\begin{aligned}
\because & H\left(\Gamma_{\mathrm{B}}\left(Z_{p^{m}}\right) ; x\right)=p^{m} q+\left(\frac{1}{2}[2 q(m p-m+p)-(m+1) p+m] p^{m-1}-\right. \\
& \left.\frac{1}{2} p^{\left\lfloor\frac{m}{2}\right]}\right) x+\left(\frac { 1 } { 2 } \left[2 q\left(p^{m+1} q-m p-2 p+m\right)+(m+1) p-\right.\right. \\
& \left.m] p^{m-1}+\frac{1}{2} p^{\left\lfloor\frac{m}{2}\right]}\right) x^{2} \\
\therefore & H\left(\Gamma_{B}\left(Z_{18}\right) ; x\right)=18+30 x+123 x^{2} . \\
\because W\left(\Gamma_{\mathrm{B}}\left(Z_{p^{m}}\right)\right)= & \frac{1}{2}\left[2 q\left(p^{m+1} q-m p-2 p+m\right)+(m+1) p-m\right] p^{m-1}+\frac{1}{2} p^{\left.\mid \frac{m}{2}\right\rfloor .} \\
\therefore & \mathrm{W}\left(\Gamma_{\mathrm{B}}\left(Z_{18}\right)\right)=276 .
\end{aligned}
$$

\section{Theorem 3.3: (Coloring of graph $\Gamma_{B}\left(Z_{p^{m} q}\right)$ ).}

A- Chromatic number of vertices of the graph

$$
\Gamma_{\mathrm{B}}\left(\mathrm{Z}_{p^{m} q}\right)=\left\{\begin{array}{l}
p^{\frac{m-1}{2}}+2, m \text { is an odd. } \\
p^{\frac{m}{2}}+1, m \text { is an even. }
\end{array}\right.
$$

B- Chromatic number of edges of the graph $\Gamma_{\mathrm{B}}\left(Z_{p^{m} q}\right)$ is $p^{m} q-1$.

Proof: A- Case 1: if $m$ is an even:

From the Theorem (2.5-A-1). Since the subgraph $k_{p^{\frac{m-1}{2}}+1}$ is the largest complete subgraph exist in the graph $\Gamma_{\mathrm{B}}\left(Z_{p^{m}}\right)$ (when $m$ is an odd). It is also clear that the number $p^{m} q$ product of multiplication the number $p^{m}$ or one of its complications in the number $q$ or one of its complications thus a new vertex will be added to the complete graph $k_{p} \frac{m-1}{2}+1 \quad$ so we have the complete graph $k_{p^{\frac{m-1}{2}}+2}$ is the largest complete subgraph exist in the graph $\Gamma_{\mathrm{B}}\left(Z_{p^{m} q}\right)$ hence the chromatic number of the graph $\Gamma_{\mathrm{B}}\left(Z_{p^{m} q}\right)$ is $\left(p^{\frac{m-1}{2}}+2\right)[7]$.

A- Case 2: if $m$ is an even:

From the Theorem (2.5-A-2). Since the subgraph $k_{p^{\frac{m}{2}}}$ is the largest complete subgraph, exist in the graph $\Gamma_{\mathrm{B}}\left(Z_{p^{m}}\right)$ (when $m$ is an even). It is also clear that the number $p^{m} q$ product of multiplication the number $p^{m}$ or one of its complications in the number $q$ or one of its complications thus a new vertex will be added to the complete graph $k_{p^{\frac{m}{2}}}$ so we have the complete graph $k_{p^{\frac{m}{2}}+1}$ is the largest complete subgraph exist in the graph $\Gamma_{\mathrm{B}}\left(Z_{p^{m} q}\right)$ hence the chromatic number of the graph $\Gamma_{\mathrm{B}}\left(Z_{p^{m} q}\right)$ is $\left(p^{\frac{m}{2}}+1\right)$ [7]. 
B- From the Lemma (2.1) so it is the vertex (0) connect with every vertex the graph $\Gamma_{\mathrm{B}}\left(Z_{p^{m} q}\right)$ then the degree of the vertex $(0)$ is $\left(p^{m} q-1\right)$ so it is the chromatic number of the edges is $\left(p^{m} q-1\right)$.

Theorem 3.4: The graph $\Gamma_{\mathrm{B}}\left(Z_{p^{m} q}\right)$ contains a subgraph that is homeomorphic to $k_{\left(p^{m}-1\right), q}$ and $k_{\left(p . q-p^{m-1}\right), p^{m-1}}$.

Proof: The first part, since the multiplying the number $p^{m}$ or one of its complications $\left(2 p^{m}, 3 p^{m}, \ldots,(q-1) \cdot p^{m}, p^{m} q=0\right)$ by number $q$ or one of its complications $\left(2 q, 3 q, \ldots,\left(p^{m}-1\right) \cdot q, p^{m} q=0\right)$ be the product $p^{m} q$ or one a complications of the number $p^{m} q$ which is equal to (0) in the ring $Z_{p^{m}} q$. Thus the graph $\Gamma_{\mathrm{B}}\left(Z_{p^{m} q}\right)$ contains a subgraph homeomorphic complete bipartite graph $k_{\left(p^{m}-1\right), q}$, as in the Figure (3.1).

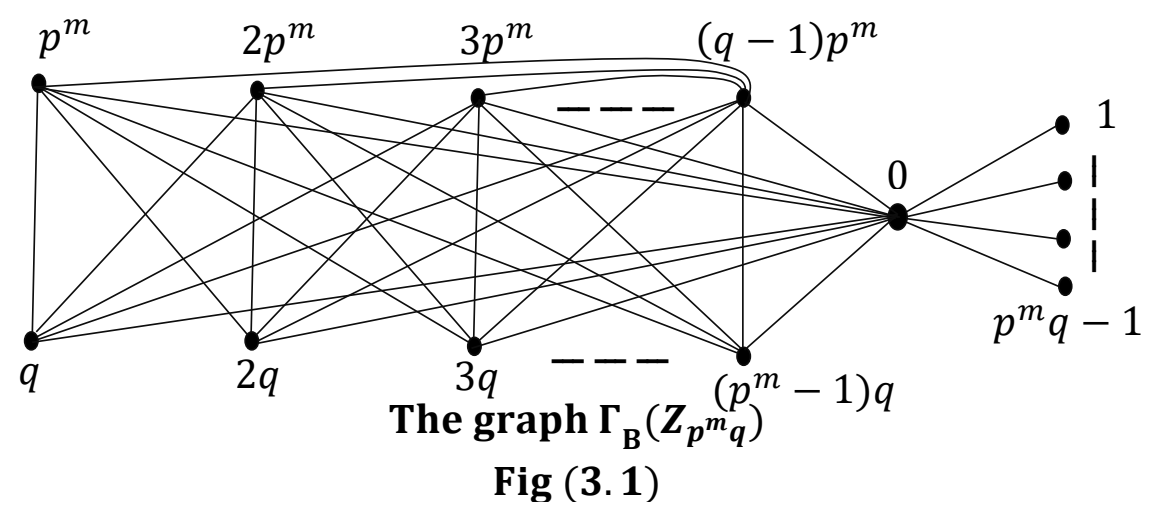

The second part, since the multiplying the number $p q$ or one of its complications $\left(2 p q, 3 p q, \ldots,\left(p^{m-1}-1\right) p q, p^{m} q=0\right)$ by number $p^{m-1}$ or one of its complications $\left(2 p^{m-1}, 3 p^{m-1}, \ldots,(p q-1) p^{m-1}, p^{m} q=0\right)$ be the product $p^{m} q$ or one a complications of the number $p^{m} q$ which is equal to (0) in the ring $Z_{p^{m}} q$. Thus the graph $\Gamma_{\mathrm{B}}\left(Z_{p^{m} q}\right)$ contains a subgraph homeomorphic complete bipartite graph $k_{\left(p q-p^{m-1}\right), p^{m-1}}$, as in the Figure (3.2).

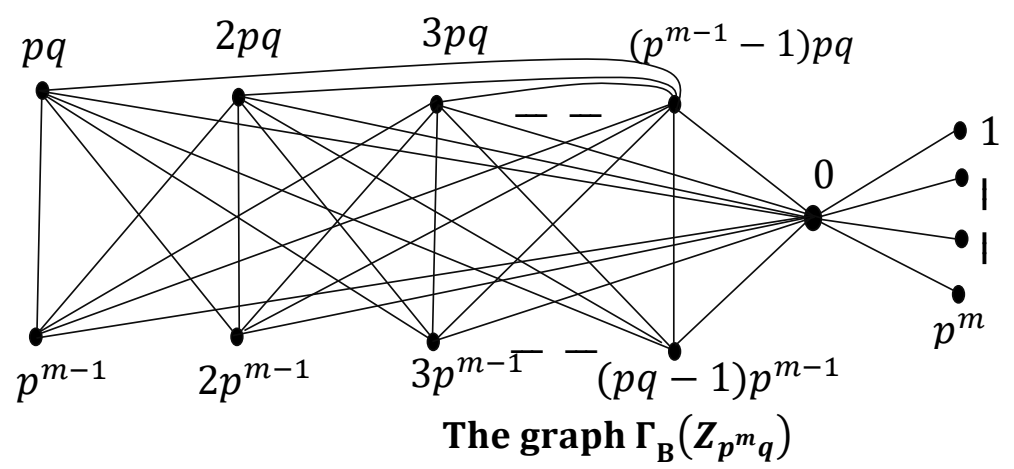

\section{Remark:}

Fig (3.2)

From the Theorem (3.4), the only graphs of the formula $\Gamma_{\mathrm{B}}\left(Z_{p^{m}}\right)$ when $q=2$ and $m=1$ does not contain a subgraph homeomorphic $k_{3,3}$ or $k_{5}$ therefore it is planar and colorable for faces. Otherwise, the graphs of the formula $\Gamma_{\mathrm{B}}\left(Z_{p^{m}}\right)$ contain a subgraph homeomorphic $k_{3,3}$ or $k_{5}$ therefore it is not planar graphs by kuratowski's Theorem.

Example 4: The chromatic number of the graphs $\Gamma_{B}\left(Z_{18}\right)$ and $\Gamma_{B}\left(Z_{22}\right)$ 
The graph is clear $\Gamma_{\mathrm{B}}\left(Z_{18}\right)$ of formula $\Gamma_{\mathrm{B}}\left(Z_{p^{m}}\right)$, where $p=3, q=2$ and $m=2$ and the graph is clear $\Gamma_{\mathrm{B}}\left(Z_{22}\right)$ of formula $\Gamma_{\mathrm{B}}\left(Z_{p^{m} q}\right)$, where $p=11, q=2$ and $m=1$.

The chromatic number of vertices of the graph $\Gamma_{\mathrm{B}}\left(Z_{p^{m}}\right)$ is $p^{\frac{m}{2}}+1$ (when $m$ is an even).

$\therefore X\left(\Gamma_{\mathrm{B}}\left(Z_{18}\right)\right)=4$.

The chromatic number of edges the graph $\Gamma_{\mathrm{B}}\left(Z_{p^{m} q}\right)$ is $p^{m} q-1$.

$\therefore \bar{x}\left(\Gamma_{\mathrm{B}}\left(Z_{18}\right)\right)=17$.

From Theorem (3.4) we get the graph $\Gamma_{\mathrm{B}}\left(Z_{18}\right)$ contains a subgraph that is homeomorphic to $k_{3,3}$ then the graph $\Gamma_{\mathrm{B}}\left(Z_{18}\right)$ it is not planar by kuratowski's Theorem.

The chromatic number of vertices the graph $\Gamma_{\mathrm{B}}\left(Z_{p^{m} q}\right)$ is $p^{\frac{m-1}{2}}+2$ (when $m$ is an odd). $\therefore x\left(\Gamma_{\mathrm{B}}\left(Z_{22}\right)\right)=3$.

The chromatic number of edges the graph $\Gamma_{\mathrm{B}}\left(Z_{p^{m}}\right)$ is $p^{m} q-1$.

$\therefore \bar{\chi}\left(\Gamma_{\mathrm{B}}\left(Z_{22}\right)\right)=21$.

From Theorem (3.4) we get the graph $\Gamma_{\mathrm{B}}\left(Z_{22}\right)$ contains a subgraph that is homeomorphic to $k_{10,2}$ it is the largest complete bipartite graph there is in the graph $\Gamma_{\mathrm{B}}\left(Z_{22}\right)$ then the graph $\Gamma_{\mathrm{B}}\left(Z_{22}\right)$ it is planar by kuratowski's Theorem.

$\therefore f\left(\Gamma_{\mathrm{B}}\left(Z_{22}\right)\right)=3$. 


\section{REFERNCES}

[1] Ahmdi M. R. and Nezhad R. J., (2011), "Energy and Wiener Index of ZeroDivisor Graphs", Iranian Journal of Mathematical Chemistry, Vol. 2, No. 1, 4551.

[2] Anderson D. and Livingston P., (1999), "The Zero-Divisor Graph of a Commutative Rings", J. Algebra, 217, 434-447.

[3] Anderson D. and Naseer M., (1993), "Beck's Coloring of a Commutative Rings", J. Algebra 159-500-514.

[4] Anna D., (2006), "Proper Colorings and p-Partite Structures of the Zero-Divisor Graph".

[5] Beck I., (1988), "Coloring of Commutative Rings", J. Algebra 116, 208-226.

[6] Burton D. M., (1967), "Introduction to Modern Abstract Algebra", University of New Hampshire.

[7] Chartrand, G. and Lesniak, L. "Graphs and Digraphs", 6th ed., Wadsworth and Brooks/ Cole. California. 2016.

[8] Hosoya H., (1985), "On Some Counting Polynomials in Chemistry", Discrete Applied Math. Vol. 19, 239-257.

[9] Husam. Q. M and Mohammad. N. A, (2018), "Hosoya polynomial and Wiener index of zero-divisor graph of Zn", Al-Rafidain Journal of Computer Sciences and Mathematics.

[10] Luma. A. K, (2019), "The Maximal Degree of Zero- Divisor-Graph of $Z_{p^{m}} q^{2}$ and $Z_{p^{m}}{ }_{q r}$, Ph.D. Thesis, Mosul University, Mosul.

[11] Narmin. J. K, (2019), "Pseudo-Von Neumann Regular Graph of Commutative Rings with some Properties of it's Graphs", M.Sc. Thesis, Tikrit University, Tikrit.

[12] Wiener H., (1947), "Structural Determination of the Paraffin Boiling Points", J. Am. Chem. Soc. 6917-20. 